\title{
MS18-P10 | Structural modification Of Perovskites by tAILOREd ExSOlution for enhanced Catalytic Activity
}

Rameshan, Raffael (Technische Universität Wien, Wien, AUT); Rameshan, Christoph (Technische Universität Wien, Wien, AUT); Ruh, Thomas (Technische Universität Wien, Wien, AUT); Nenning, Andreas (Technische Universität Wien, Wien, AUT); Opitz, Alexander (Technische Universität Wien, Wien, AUT); Rameshan, Christoph (Technische Universität Wien, Wien, AUT); Rameshan, Raffael (Technische Universität Wien, Vienna, AUT)

Perovskites provide a dynamic structure, where induced oxygen vacancies can trigger the formation of nanoparticles on the surface. The process of nanoparticle formation can thus be controlled and reversed by choosing a suitable chemical potential of the gas phase or by polarization (i.e. applying voltage to the system) o the perovskite.

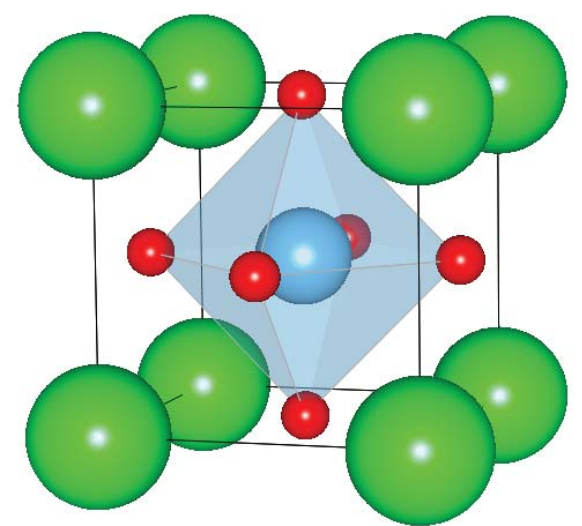

Using a lab-based near ambient pressure X-ray photoelectron spectrometer (NAP-XPS) that is specially designed for investigations of electro-catalytic systems under realistic operating conditions combined with in-situ $\mathrm{X}$-ray diffraction spectroscopy (XRD), scanning electron microscopy (SEM) and energy dispersive X-ray spectroscopy (EDX) we provide first results on the effect of exsolution on water gas shift (WGS) and reverse WGS reaction.

Acknowledgement. This work has received funding from the European Research Council (ERC) under the European Union's Horizon 2020 research and innovation programme (grant agreement n 755744 / ERC - Starting Grant TUCAS).

[1] K. Momma and F. Izumi, "VESTA 3 for three-dimensional visualization of crystal, volumetric and morphology data," J. Appl. Crsallogr., 44, 1272-1276 (2011).

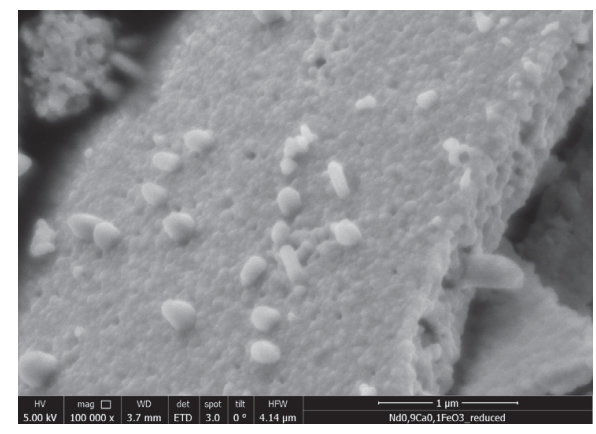

